\title{
Empirical Power Consumption Model for UAVs
}

\author{
Hasini Viranga Abeywickrama, Beeshanga Abewardana Jayawickrama, Ying He, Eryk Dutkiewicz \\ University of Technology Sydney, Global Big Data Technologies Centre \\ Email: $\{$ Hasini.V.Abeywickrama\}@student.uts.edu.au \\ \{Beeshanga.Jayawickrama, Ying.He, Eryk.Dutkiewicz\}@uts.edu.au
}

\begin{abstract}
Unmanned Aerial Vehicles (UAV) are gaining popularity in a range of areas and are already being used for a wide variety of purposes. While UAVs have many desirable features, limited battery lifetime is identified as a key restriction in UAV applications. Typical UAVs being electric devices, powered by on-board batteries, this constrain has limited their capabilities to a considerable extent. Thus planning UAV missions in an energy efficient manner is of utmost importance. To achieve this, for prediction of power consumption, it is necessary to have a reliable power consumption model. In this paper, we present a consistent and complete power consumption model for UAVs based on empirical studies of battery usage for various UAV activities. The power consumption model presented in this paper can be readily used for energy efficient UAV mission planning.

Index Terms-UAV, power consumption, energy consumption, power consumption model
\end{abstract}

\section{INTRODUCTION}

Unmanned Aerial Vehicles (UAV) are fast becoming a popular choice for a wide variety of commercial applications. Their many desirous features like the ability to hover, the ability to fly over dangerous and inaccessible areas and ease of deployment enable UAVs to be widely used in many aspects of social life [1].

Despite the increasing popularity, a number of challenges hinder the full potential of UAV applications. Limited battery lifetime is identified as a key drawback in UAVs [2]. Typical UAVs are electric vehicles that are powered by on-board batteries which have limited lifetime. Due to this, most UAV applications are not able to reach their full potential.

In efforts to overcome the limited flight time of UAVs caused by limited battery lifetime, it is important to plan the UAV missions in such a way that UAV power consumption is minimal. The UAV trajectories should be planned in an energy efficient manner. To achieve energy efficient UAV missions, it is important to identify and minimize the actions that are high power consuming. For this, it is highly necessary to follow a reliable and complete power consumption model for UAVs. An accurate model of battery performance in different scenarios would allow further flight mission planning and recharging optimization for UAVs [2].

In this paper we present a reliable power consumption model for UAVs, that profiles the power needs of commonly used UAV actions. The presented power consumption model is based on empirical studies, which will be described in detail later in this paper.

In literature there appears to be limited empirical studies of battery performance of UAVs, in contrary to ground electric vehicles [2]. The authors of [3] have performed brief experiments and state the power consumption of a few basic UAV maneuvering actions. In [2], the authors have conducted more detailed studies on UAV battery performance. However, both [2] and [3] cannot be considered complete power models for UAVs as they only consider a limited set of flight scenarios and they do not contain enough information for comparisons or power predictions. To the best of our knowledge, work presented in this paper is the first power model on Intel Aero Ready to Fly Drone [4], the method we have used could be used on other UAVs for similar applications. The power model presented in this paper can be readily used for energy efficient UAV path planning and obstacle avoidance purposes.

Modelling and predicting power consumption of ground electric vehicles have been a popular area of study. One such method of modelling is the white-box approach, where the understanding of energy consumption of electric vehicles is based on specific vehicle dynamics [5]. Another method for modelling power consumption is the black-box method. In this method a general statistical approach using regression model, without vehicle dynamics model, is used to model the power consumption of vehicles [6]. The black-box approach is more convenient and provides sufficient information for power consumption predictions for UAV missions. Hence, in the presented power consumption model we employ a blackbox approach, considering different flight conditions but not taking vehicle dynamics into account.

The rest of this paper is structured as follows. Section II gives an overview of the empirical studies of UAV power consumption. The measurement results of the studies and power consumption model are presented in Section III. Section IV discusses the challenges encountered when conducting the empirical studies. Section V concludes the paper.

\section{OVERVIEW OF THE EMPIRICAL STUdy OF UAV POWER CONSUMPTION}

In order to come up with a reliable power consumption model for UAVs, we conducted a series of empirical studies. These studies were focused on understanding the influence of several factors on UAV power consumption. The factors taken into consideration are listed below:

1) On-ground power consumption

2) Impact of communication with the ground station

3) Impact of movement - hovering, horizontal movement, vertical movement

4) Impact of speed 


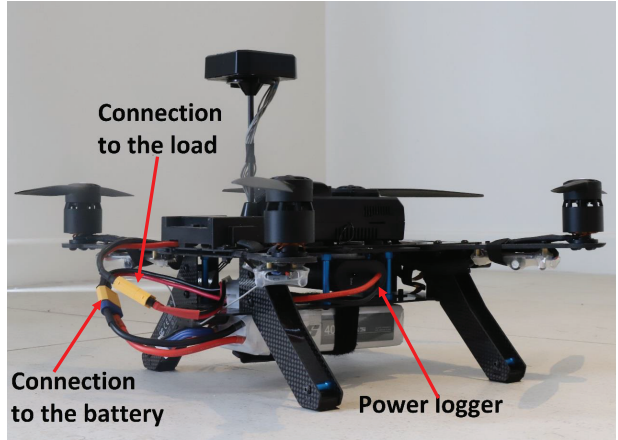

Fig. 1: Set up connecting Power Meter to UAV

5) Impact of payload

All experiments were performed on Intel Aero Ready to Fly Drone [4]. For improved accuracy, we used a Power Meter [8] that measures current, voltage and power consumption. We further used a Data Adaptor [9] to reliably retrieve the data recorded by the Power Meter. During the studies where the data logging interval of the Power Meter was not sufficient for the preferred accuracy, we manually recorded the real time data displayed taking the Power Meter off-board.

The setup of connecting the Power Meter to the UAV is shown in Figure 1.

All the experiments were performed in obstacle free outdoor environments where wind was minimal.

\section{Measurement Results and Power Consumption MODEL}

The results of the experiments are described below.

\section{A. On-ground power consumption}

We considered two basic scenarios to understand the UAV power consumption when it is on-ground.

1) Idle mode: The UAV was powered on and kept on the ground without the propellers rotating and with no contact with the ground station. In this scenario, the UAV would need power to broadcast Wi-Fi signal, for LED indicators and internal processing. The instantiations power consumption was logged over a period of 20 minutes. The power consumption over time is shown by the blue line in Figure 2.

It is seen that the power consumption in the idle mode is stable over time. The average power consumption is $8.2412 \mathrm{~W}$ with a standard deviation of $0.0487 \mathrm{~W}$.

2) Armed mode: The same experiment was carried out again with propellers of the UAV set to rotate. In this scenario, the UAV would need power for the rotation of propellers, in addition to the energy needs of the previous mode.

The power consumption for this scenario is shown by the red line in Figure 2. There is an initial spike in power consumption, due to the high draw of current when starting four motors. The power consumption stabilises over time. Once stabilized, the average power consumption is $27.7748 \mathrm{~W}$ with a standard deviation of $0.1256 \mathrm{~W}$.

From the above results, it can be noted that the average power consumption in the armed mode is more than three

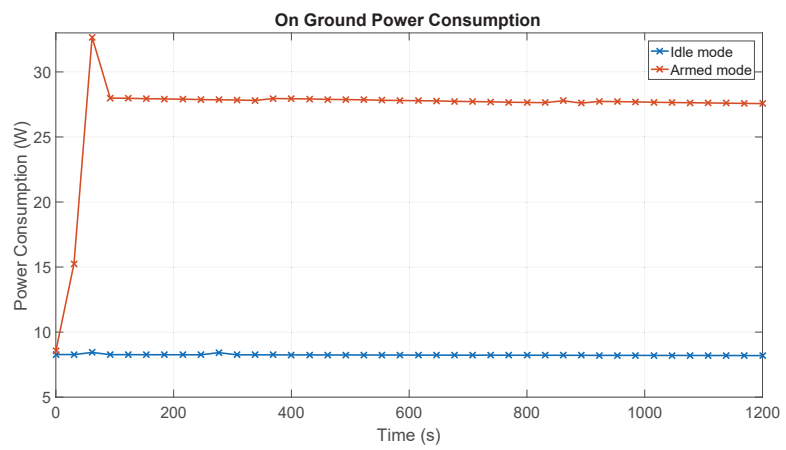

Fig. 2: On-ground Power Consumption of the UAV

times higher than that of idle mode. Thus, it can be concluded that the motors for rotating propellers consume considerably high power. It would be advantageous energy-wise, to develop energy efficient motors for UAVs.

\section{B. Impact of communication with the ground station}

We conducted experiments to understand the power needs of the UAV for Wi-Fi communication and to communicate Global Positioning System (GPS) data with the ground station.

The studies in this section were performed in the 'idle mode' mentioned in Section A Subsection 1. The power measurements shown in this section are equal to the total power consumption of the UAV in the given scenarios, which is the sum of the power consumption discussed in Section A Subsection 1 and power consumption for communication that is stated below.

1) Wi-Fi communication: The UAV was powered and set to maintain Wi-Fi communication with the ground station (with no GPS lock). When flying UAVs indoors, most often the only mode of communication between the ground station and the UAV is Wi-Fi. Thus, it is important to understand the power requirement for Wi-Fi communication.

The ground station used in this study was a QGroundControl installed laptop. In this study we used the 'node mode' on the UAV and the ground station as an Access Point (AP). The UAV would share its calibration information, status information (armed, disarmed, landed etc.), battery information and basic set up parameters including take off altitude, speed etc. with the ground station using Wi-Fi communication.

The ground station and the UAV had clear line-of-sight with no obstacles in between. The distance between UAV and ground station was gradually increased. The instantaneous power in each point was recorded over a period of 20 minutes and the average power consumption for each point was taken to plot the blue graph in Figure 3.

It can be seen that the total power consumption with WiFi communication remains stable over the distance considered (up to $50 \mathrm{~m}$ ). It has an average of $8.2618 \mathrm{~W}$ and a standard deviation of $0.0123 \mathrm{~W}$.

2) GPS communication: The same process as above was carried out, with UAV maintaining GPS communication with the ground station. Since GPS is imperative for outdoor missions, understanding its power requirement is important. 


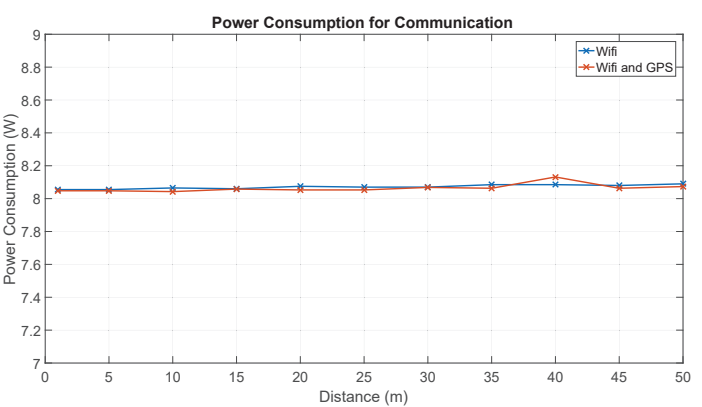

Fig. 3: Power Consumption for Communication

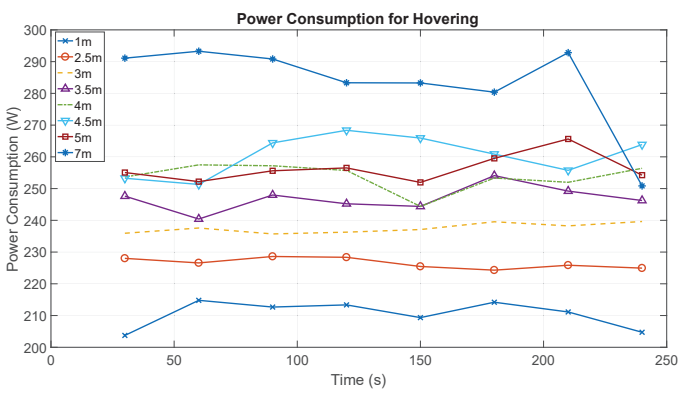

Fig. 4: Impact of Hovering Altitude on Power Consumption

The result of the study is shown in red graph in Figure 3. It can be seen that the total power consumption with GPS communication does not have a considerable variation over the distance considered. The average total power consumption is $8.2637 \mathrm{~W}$ with a standard deviation of $0.0243 \mathrm{~W}$.

It can be concluded that in comparison to the idle mode power consumption stated in Section A Subsection 1, the power consumption for communication is negligible. Thus in flight scenarios where the UAV is no further than $50 \mathrm{~m}$ from the ground station, the power consumption for communication can be considered negligible.

\section{Impact of movement - hovering, horizontal movement, vertical movement}

The power measurements shown in this section are the total power consumed by the UAV in performing the stated actions. This includes power for basic arming mentioned in Section A Subsection 2, power for communication mentioned in Section $B$ and the power for movements discussed in this section.

1) Hovering: The UAV was made to hover at different altitudes to understand the behaviour of power consumption while hovering and the effect of hovering altitude. It was noted that the power consumption remains reasonably constant over the period of hovering, except for minor fluctuations due to instability (Figure 4).

It can also be seen that the power requirement for hovering increases with altitude. Figure 5 shows the best fitting trend of power increase with altitude. The equation of power trend when hovering is given by (1), where $P$ is the power requirement in watts and $H$ is the relative hovering altitude in meters.

$$
P=13.0397 H+196.8490
$$

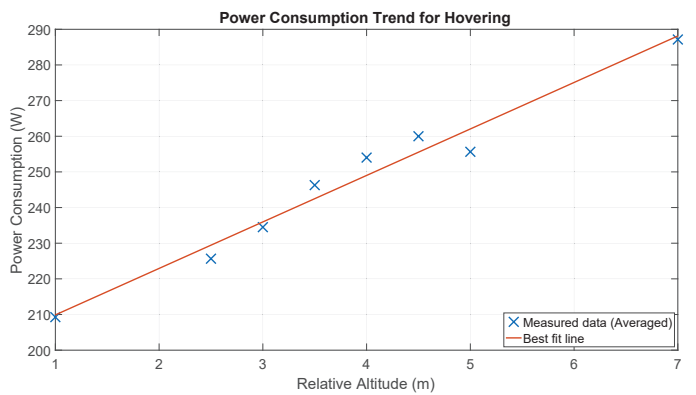

Fig. 5: Power Consumption Trend for Hovering with Altitude

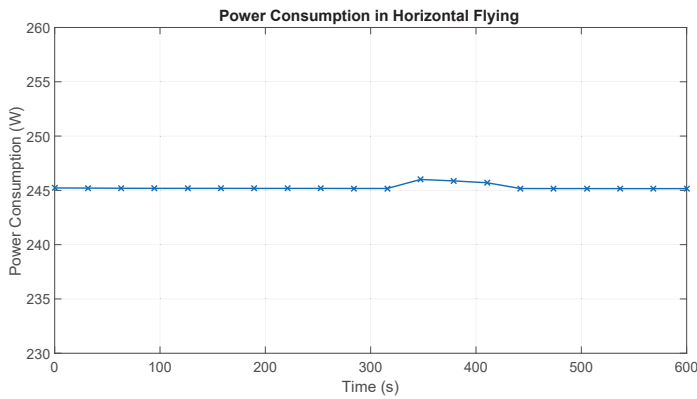

Fig. 6: Power Consumption for Horizontal Flying

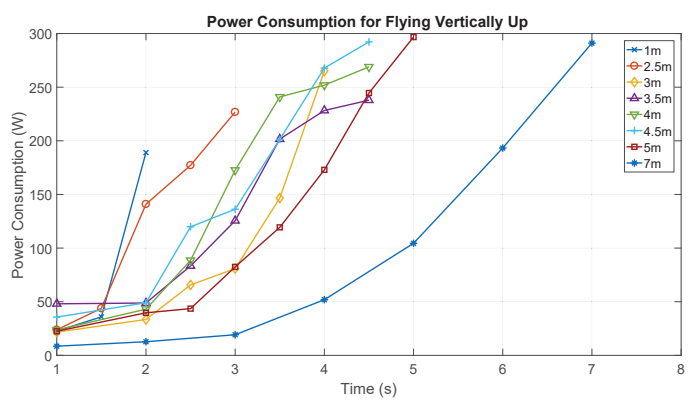

Fig. 7: Power Consumption for Flying Vertically Up

2) Horizontal movement: The UAV was made to fly in a straight horizontal line of a relative altitude of $3 \mathrm{~m}$, for a period of 10 minutes. The instantaneous power consumption over this period is plotted in Figure 6 .

It can be seen that the power consumption remains consistent throughout the flight period. There are slight fluctuations in power consumption due to minor vertical movements the UAV made. The average power consumption for horizontal flying is $245.2815 \mathrm{~W}$ with a standard deviation of $0.2540 \mathrm{~W}$.

3) Vertical movement: The UAV was made to take-off and fly vertically to reach different altitudes. All these studies were performed with a take-off velocity of $1 \mathrm{~m} / \mathrm{s}$. The power taken to vertically fly to reach different altitudes in shown in Figure 7. It can be noted that the power consumption for flying increases with the altitude.

It is often useful to have an idea of the total energy consumption of an activity, in addition to the instantaneous power readings. The total energy consumption for vertical flying can be calculated by the integral of power over the period of vertical flying. This is shown in (2), where $E$ is the energy consumption for vertical flying, in joules. $P$ is the instantaneous power consumption for vertical flying in watts. $t$ is the time interval of the flight in seconds. 


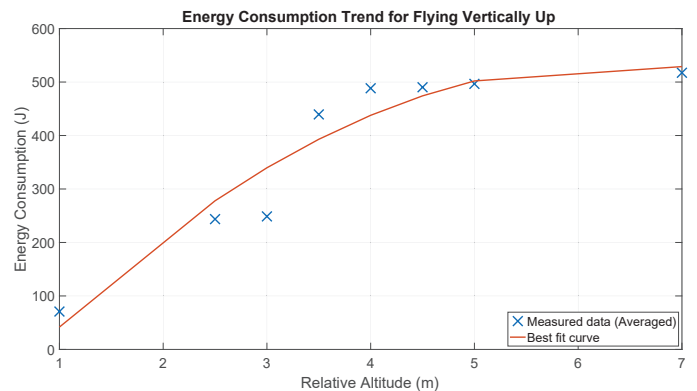

Fig. 8: Energy Consumption for Flying Vertical Up

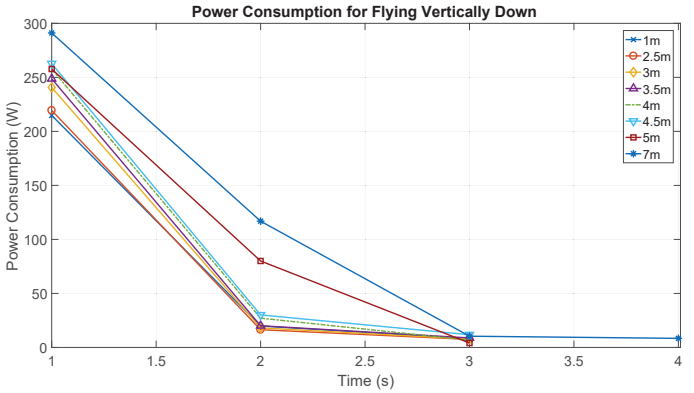

Fig. 9: Power Consumption for Flying Vertically Down

$$
E=\int_{0}^{t} P d t
$$

The right hand side of the above equation is also equal to the area under respective curves shown in Figure 7.

From Figure 7, it can be noted that the power requirement for vertical flying correlates the overall vertical flight distance. Consequently, the energy requirement to fly up to a level depends on the vertical distance that needs to be travelled. Figure 8 shows the best fitting relationship between the energy requirement and vertical flying distance. The relationship shown in Figure 8 is given by the equation below, where $\mathrm{E}$ is the total energy to fly vertically up to reach a certain relative altitude in joules, $\mathrm{H}$ is the relative altitude that needs to be reached in meters.

$$
E=-16.9396 H^{2}+216.6944 H-157.9473
$$

We conducted the same experiment, making the UAV fly vertically down. The UAV was stationed at different altitudes and made to fly vertically down and land on ground. The instantaneous power consumption for each altitude scenario is shown in Figure 9. And the best fitting relationship between the distance to fly vertically down and the energy consumption is shown in Figure 10.

The relationship shown in Figure 10 is given by (4), where $E$ is the energy in joules and $D$ is the distance to fly vertically down in meters.

$$
E=4.6817 D^{2}-11.9708 D+135.3118
$$

\section{Impact of speed}

We conducted studies to understand the impact of speed on UAV power consumption. We made the UAV fly vertically up for $3 \mathrm{~m}$ in different speeds. The instantaneous power consumption for each speed scenario is shown in Figure 11.

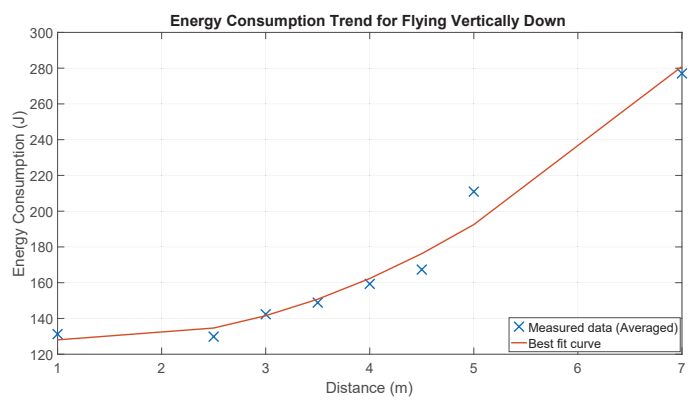

Fig. 10: Energy Consumption Trend for Flying Vertically Down

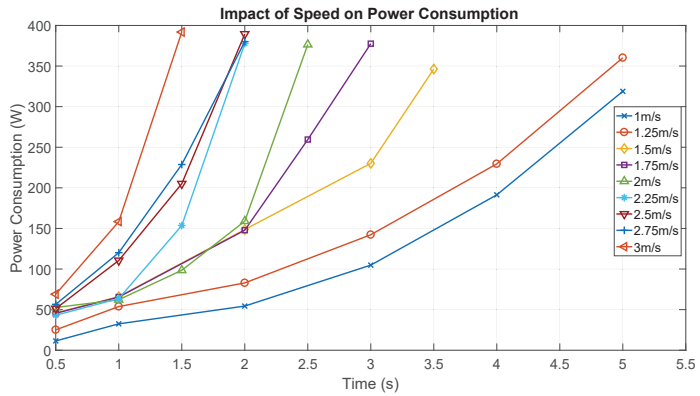

Fig. 11: Impact of Speed on Power Consumption

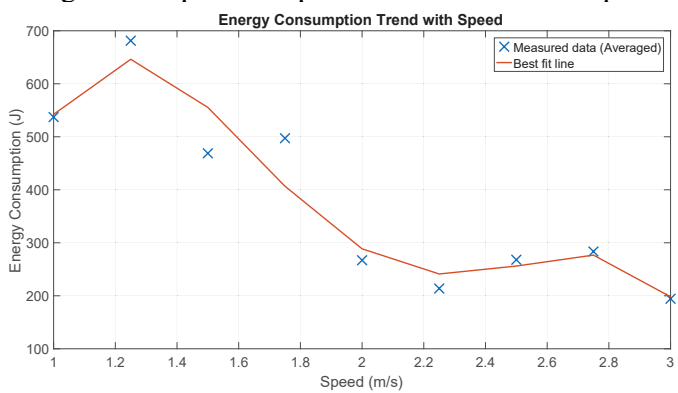

Fig. 12: Energy Consumption Trend with Speed

It can be seen from Figure 11, that the instantaneous power consumption increases considerably with the speed. As mentioned previously in this paper, the energy consumption of each scenario can be obtained by the area under the curves in Figure 11. A direct relationship between the area under curves and speed cannot be seen. Although the instantaneous power increases with speed, since the total time taken to reach a certain height decreases, the total energy consumption does not directly increase with speed.

The best fitting relationship between speed and energy consumption is shown in Figure 12.

The relationship between energy consumption and speed (Figure 12) is given by (5), where $E$ is the energy consumption in joules and $V$ is the speed of the UAV in meters per second.

$E=-0.0516 V^{4}+0.4298 V^{3}-1.2804 V^{2}+1.5816 V-0.6251$

\section{E. Impact of payload}

The UAV was mounted with different payloads and made to hover at a relative altitude of $2.5 \mathrm{~m}$. Although Intel Aero drone's maximum gross take-off weight is 1900g [4], under the environmental conditions the studies were carried out, the UAV was only able to handle up to a take-off weight of $1600 \mathrm{~g}$ 


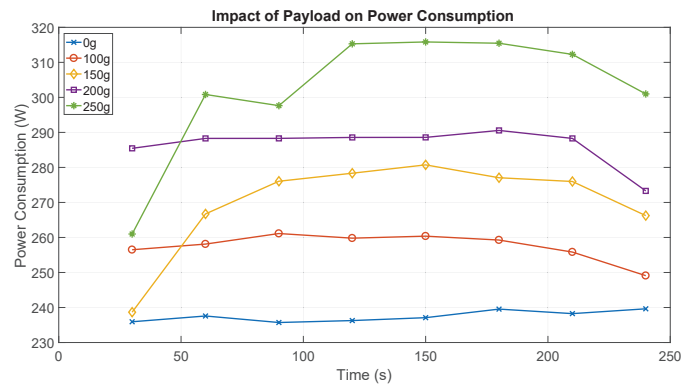

Fig. 13: Impact of Payload on Power Consumption.

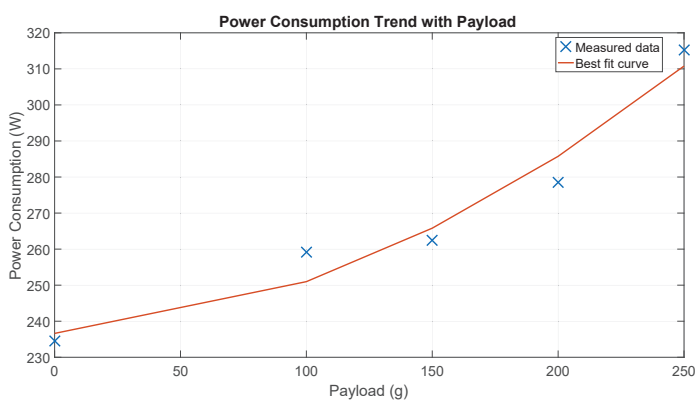

Fig. 14: Power Consumption Trend for Payload

(external payload of $250 \mathrm{~g}$ ) and steadily reach the required altitude. When mounted with $300 \mathrm{~g}$, the UAV became unstable.

The power consumption for hovering with payloads is shown in Figure 13. It can be seen that there is a clear relationship between power consumption and payload. As the payload of the UAV increases, the power consumption of the UAV increases accordingly. The best fitting relationship between the payload and power consumption is shown in Figure 14. The relationship shown in Figure 14 is given by (6), where $P$ is the power consumption in watts and $L$ is the payload in grams.

$$
\begin{gathered}
P=0.001 L^{2}+0.0416 L+236.62 \\
\text { IV. CHALLENGES }
\end{gathered}
$$

This study involved a number of challenges. The UAVs have associated limitations to the weight and the dimensions of the equipment they are capable of carrying. Most of the commercially available power loggers are either too heavy or too large in size for a UAV to carry. It was a challenge to find a commercially available power logger that is lightweight and small enough in size for Intel Aero Ready to Fly Drone to carry on-board.

To control the UAV to get the expected behaviour, strength of GPS signal is crucial. It is necessary to get a 3D GPS lock for the UAV with the visibility of 15 satellites in the minimum. Environmental conditions have a very high impact on the availability and strength of GPS signal. It is imperative to have no obstacles such as buildings or trees around the area of experiment.

When conducting experiments with payloads, it is necessary to perfectly balance the external payloads on the UAV. This is crucial for the flight and safe landing.
It is important to have favourable wind conditions. The UAV finds it difficult to take off and perform the assigned tasks in windy environments. This highly affects the power consumption of the UAV as well.

\section{CONCLUSION}

A complete and reliable power consumption model for UAVs, is highly necessary for energy efficient mission planning. It is important to identify the factors that have an influence on a UAV's power consumption, and the power requirements of commonly used UAV maneuvering actions.

In this paper we have presented an empirical power consumption model which considers on-ground power consumption, impact of communication, hovering, vertical and horizontal movements, speed and payload on a UAV's power consumption. The proposed model can be efficiently used for prediction of energy requirements and energy efficient UAV mission planning.

Employing UAVs for $5 \mathrm{G}$ communications, UAV mounted base stations to provide coverage for example, would require extensive knowledge of power consumption patterns. The model presented in this paper can be readily used for deriving the optimal height, speed and the favourable payload in such instances. UAV aided data collection in wireless sensor networks is currently being thoroughly researched. The model we propose in this paper can be used to design optimal paths for the UAVs to minimize the energy spent on flying while covering the entire sensor network. In addition, the model presented can be used to minimise the energy consumption in UAV aided delivery, coverage and surveying scenarios.

\section{REFERENCES}

[1] H. V. Abeywickrama, B. A. Jayawickrama, Y. He, E. Dutkiewicz, "Algorithm for Energy Efficient Inter-UAV Collision Avoidance." International Symposium on Communications and Information Technologies, Cairns, Australia 2017

[2] C.-M. Tseng, C.-K. Chau, K. Elbassioni, and M. Khonji. (2017) "Flight tour planning with recharging optimization for batteryoperated autonomous drones." [Online]. Available: https://arxiv.org/ abs/1703.10049

[3] S. Ahmed1, A. Mohamed, K. Harras, M. Kholief and S. Mesbah, "Energy Efficient Path Planning Techniques fo UAV-based Systems with Space Discretization." IEEE Wireless Communications and Networking Conference (WCNC), 2016.

[4] Intel Australia. (2017, September.). Specifications for the Intel Aero Ready to Fly Drone [Online]. Available: https://www.intel.com.au/content/www/au/en/support/articles/ 000023272/drones/development-drones.html

[5] E. Kim, J. Lee, K. G. Shin, "Real-time prediction of battery power requirements for electric vehicles." IEEE/ACM International Conference on Cyber-Physical Systems,Philadelphia, USA 2013

[6] A. Cappiello, I. Chabini, E. K. Nam, A. Lue, M. A. Zed, "A Statistical Model of Vehicle Emissions and Fuel Consumption." IEEE 5th International Conference on Intelligent Transportation Systems, Singapore, Singapore 2002

[7] QGroundControl Home[Online] Available: http://qgroundcontrol.com/

[8] Jaycar Electronics.Digital DC Power Meter with Internal Shunt[Online] Available: https://www.jaycar.com.au/digital-dc-power-meter-withinternal-shunt/p/MS6170

[9] Jaycar Electronics.USB Data Adaptor for DC Power Meters[Online] Available: https://www.jaycar.com.au/usb-data-adaptor-for-dc-powermeters/p/MS6174 